wenn infolge der dringenden Bedürftigkeit oder der Unerfahrenheit einer Partei ein Vertrag geschlossen wurde, bei dem ein auffälliges Missverhältnis zwischen Leistung und Gegenleistung besteht. In deutschem Recht wird diese Situation in $₫ 138$ Absatz 2 BGB behandelt unter dem Stichwort ,Wucher”.

Das neue Zivilrechtsgesetzbuch hat erstmals die Persönlichkeitrechte geregelt, im Teil über die Personen.

Die Verschollenheit von Personen wurde neu geregelt, und zwar ihre Folgen im Erbrecht

Im Allgemeinen Teil des neuen ZGB wird auch unterschieden zwischen Vereinigungen und Gesellschaften.

Im Abschnitt über die Rechtsgeschäftslehre wurde die gesetzliche Terminologic der wissenschaftlichen Terminologie angepasst.

Ein wichtiger und interessanter Unterschied zwischen dem AT des CC/2002 und dem $A T$ des BGB besteht darin, dass im CC/2002, wie auch schon im CC/1916, auch die Generalklausel für die deliktische Haftung im AT geregelt ist.

Eine andere Generalklausel, die künftig eine sehr wichtige Rolle spielen könnte, stellt der Art. 187 über den Rechtsmissbrauch dar.

Meine Damen und Herren,

nochmals vielen Dank für Ihre Aufmerksamkeit und Geduld.

\section{LA COLPA E IL RISCHIO}

\section{Andrea Marighetto*}

\section{Introduzione}

L'analisi storica delle fonti romane ha, per i civilisti, un'importanza fondamentale, dal momento che permette di cogliere l'influenza romana sulla costruzione dei modelli giuridici ancora presenti nella codicistica contemporanea.

L'elaborazione delle fonti è, dunque, intesa come metodo di studio al fine di poter intendere non solo la terminologia ed $i$ concetti fondanti, ma anche per analizzare e comprendere il determinarsi del diritto attuale.

Per comprendere l'istituto della colpa, intesa quale elemento dell'illecito civile, é dunque indispensabile un'analisi del fondamento storico-giuridico del concetto stesso di illecito civile, e viceversa: colpa e responsabilità, infatti, appaiono da sempre strettamente connessi e complementari.

Il presente studio, oltre a prendere in esame l'evoluzione storica del concetto di colpa e dell'istituto stesso della responsabilità civile, persegue lo scopo di analizzare come l'elemento della colpa abbia, dunque, influenzato e determinato le dottrine relative alla responsabilità civile.

Al tradizionale principio colpevolistico della responsabilità, si sono quindi affiancate le teorie sul rischio profitto e sul rischio creato, che richiamano prepotentemente i meccanismi della responsabilità oggettiva, o della cosiddetta responsabilità "senza colpa". Si assiste, in questo modo, ad uno spostamento, o meglio, ad una coesistenza sempre più considerevole tra i diversi criteri di imputazione dell'illecito.

L'evoluzione industriale, l'aumento dei consumi hanno determinato un corrispondente mutamento della società ove il singolo individuo viene ad essere maggiormente esposto ai rischi di una commercializzazione esasperata ove l'interesse economico del produttore tende a sovrapporsi alla protezione del singolo e, comunque, della collettività. Da qui, lo sviluppo di teorie, del cosiddetto socialismo giuridico, che appunto prendono in considerazione siffatta asimmetria, e propongono una rivisitazione de tradizionale diktat Jheringhiano del nessuna responsabilità senza colpa. Si assiste, quindi, ad un affiancamento sempre meno eccezionale del criterio di imputazione oggettiva della responsabilità, e sempre più ad una sua applicazione generalizzata.

Il presente studio persegue anche la finalità di evidenziare come i sistemi di diritto da

Laurea in Dirtto con PUniversità di Verona (Italia). Master in relazion minternazionali con l'Università di Padova (Italia) Specialista in Alternative Dispute Resolution in San Diego (Stati Uniti). Specialista in Diritto della Integrazione con Organizzazione degi Stati Americani (Brasile). Specializzando in Diritto del Consumatore e Dintti Fondamentali con lUFRGS Brasile) Dollorado in Dirito per 1'UFRGS (Brasile). 
sempre considerati più tradizionali e quelli più innovativi hanno dunque accolto queste teorie. Interessante è, dunque, osservare come, in realtà, le principali codificazioni della famiglia di civil law hanno, per lo più, mantenuto il criterio colpevolistico come criterio principale di imputazione dell'illecito. Al più, la colpa è stata, per così dire, maggiormente oggettivizzata per esempio attraverso quelle ipotesi di presunzione di colpevolezza in cui "la prova contraria" si è ridotta a poche improbabili fattispecie. Si è dunque, per la maggior parte dei casi, preferito affiancare alle tradizionali codificazioni, previsioni legislative speciali, aventi ad oggetto il ricorso all'utilizzo della responsabilità oggettiva.

I - Dalla responsabilità per colpa alla responsabilità oggettiva, quale strumento di razionalizzazione del mercato, solidarietà sociale e dominazione dell'attività $\mathrm{d}$ impresa nella società del benessere.

\section{A) Evoluzione storico-giuridica del concetto "romano" di responsabilità civile}

\section{1) Considerazioni generali sulla "teoria classica della colpa"}

La questione relativa alla classicità della colpa quale elemento dell'illecito aquiliano suggerisce un breve excursus storico-giuridico sui fondamenti della stessa responsabilità civile.

Il giurista italiano Guido Alpa, nota come la terminologia classica non riguardasse tanto la responsabilità, quanto, piuttosto, il damnum e l'iniuria, ove con il termine iniuria -e ciò appare estremamente rilevante ai fini del presente studio, per i motivi che verranno esaminati in seguito - si intendeva soprattutto "ciò che era stato fatto non iure o contra ius".

Ma analizziamo con calma. All'epoca delle XII 'Tavole ${ }^{3}$, vi erano vari tipi di iniuria $a^{4} \mathrm{e}$ ciascuna di esse rappresentava una fattispecie tipica. Solo dall'età classica, fu possibile individuare i primi tentativi di realizzazione di una regola generale sul danno e sulla sanzione penale: è proprio di questa epoca l'actio legis A quilia' con la quale, per la prima volta, si cercò di generalizzare

ALPA, Guido, Diritto della responsabilità civile, Roma-Bari: Ed. Laterza, 2003 p. 44 ne IRTI, Natalino (Collana diretta da) Istituzioni di Diritto. Serie monografica.

CANNATA, Carlo Augusto. Sul testo della Lex Aquilia e la sua portata originaria, in VACCA, Letizia. La responsabilità civile da atto illecito nella prospettiva storico-comparatistica, I Congresso internazionale ARISTEC, Madrid, 7-10 ottobre 1993 Torino: Giappichelli, 1995 p. 36 come riportato da ALPA, Guido, Op. cti. p. 78

La Lex XII Tabulanum costitui il nucleo del ius legitimum vetus. Fu emanata nel $451-450$ a. C.. Tale lex ha lindubbio merito di aver concesso una sorta di trasparenza delle procedure necessarie per accertare o tutelare i propri diritti (condizione di minglicramento della plebe): con l'introduzione delie legis actiones s1 1 introdusse un rigido formalismo che importava perle part in lite il rigoroso e imprescindibile rispetto procedurale. In TALAMANCA, Mario. Istituzioni di Diritto Romano. Milano: Guffre 1990.

4 Letteralmente: singole ipotesi tipiche di danneggiamento.

LA Actio legis Aquiliae fu introdotta 11ell' ordinamento giuridico romano dalla lex Aquiliae de damno, agli inizi del III secolo a.C E' strutturata in tre capitoll' nel ptrmo capitolo, il soggetto che aveva ucciso ingiustamente uro schiavo ovvero un quadrupede

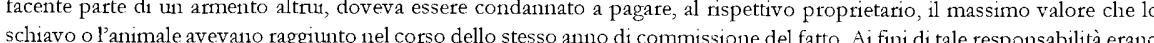

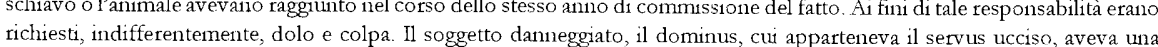
doppia possibilità: o poteva accusare l'omicida della commissione del crimine intentato una esigere il risarcimento del danno subito, agendo "in sede civile" in forza della lex Aquilia. Nel secondo capitolo, era prevista l'ipotesi di acceptilatio (quando l'adstipulator, in frode allo stipulans principale, accreditava sul proprio libro giomale della un'azione penale e, allo stesso tempo, un'azione di carattere reipersecutorio, e dunque risarcitorio, con cui il danneggiato poteva rivalersi sul danneggiante ${ }^{6}$.

Come anche evidenziato dal giurista brasiliano Alvino Lima ${ }^{7}$, l'ultimo capitolo dell a legge (si confronti nota 5) rappresenta la parte più importante della legge stessa, in quanto viene definito il concetto di damnum iniuria datum su cui i giureconsulti di epoca classica, così come poi i pretori, contribuirono a forgiare il concetto della responsabilità civile. Tale formula, il damnum iniuria datum, ovvero tale delictum, rappresentò dunque il punto di partenza nella storia e nell'evoluzione giuridico-concettuale dell'istituto della responsabilità civile.

La lex Aquilia, nella sua versione più originale, non rappresentava de facto un'azione di carattere generale, almeno non nel senso delle modeme codificazioni di famiglia romanogermanica, atteso che la stessa prevedeva la possibilità di risarcimento per le sole determinate ipotesi (si veda in nota 5 ) in essa ben tipicizzate. Assunse solo col passare degli anni e con l'interpretazione della giurisprudenza, quella valenza di vera e propria "clausola generale" che risultò essere propria degli ordinamenti di stampo romano-germanico ${ }^{8} \mathrm{e}$, in modo particolare, del padre delle codificazioni mode rne, il Code Napoléon del $1804^{\circ}$.

Originalmente, la lex Aquilia, regolando le varie ipotesi di danneggiamento, richiedeva per la sua applicazione due requisiti fondamentali: il danneggiamento corpore et corpori e l'iniunia ${ }^{10}$ : da qui, l'interpretazione della giurisprudenza e l'allargamento a più ipotesi di danneggiamento. L'ambito di applicazione di questa legge, infatti, fu successivamente e notevolmente estes $\mathrm{a}^{11}$ : furono ricomprese tutte quelle ipotesi di damnum (danneggiamento) causato senza lo sforzo fisico - non corpore - e, la stessa lex Aquilia fu interpretata nel senso

contabilità una certa somma) sanzionata con una pena pecuniaria. Il terzo capitolo della lex Aquilia de damno, riguardava tutte ealtre fattispecie di danno inclusi quelli cagionati ingustamente a cose inanimate La legge per completerza di esposizione stabiliva inoltre che il danneggiante doveva essere condannato al pagamento non di una cifra pari al valore della res in quell'anno bensi di una cifra determinata in relazione al valore della res nei 30 gioni precedentil fatto produttivo del danno. In BURDESE, Alberto. Manuale di Diritto Privato Romano. Torino: UTET 1993, p. 529. Cfr. Gai 3.210; D.9.2.2 pr. (Gai); Gai 3.215, D. 9.2 .27 (Ulp.).

6 ALPA, Guido. La responsabilità civile nel Trattato di Diritto Civile (a cura di). Milanno: Giuffrè 1999

7 LiMA, Alvino. Culpa e Risco. Săo Paulo: RT, 1998 p. 22.

8 MAY, Gaston. Manuel de droit romainl Paris 1932, come citato da LIMA, Alvino. Op. cit

9 DAVID, René; JOUFFRET-SPINOSI, Camille. Les Grands Systèmes de droit contemporains. Paris: Dalloz, 2002, p. 89 e ss, 10 GIORDANO, Francesca. La colpa nell'illecito extracontrattuale tra vecchi e nuovi orientamenti di dottrina e giurisprudenz in Giustizia Civile, (a cura di SGROI, Vittorio). Milanı: Giuffré 1995, p. 173

11 Importante appare la ricostruzione di ALPA, Guido. Ult. Op. cit. p. 28 e ss. in cui specifica che l'oggetto della lex Aquilia non era il patrimonio, inteso come insieme dei beni costituentí la ricchezza del dominus, ma la cosa stessa nella sua materialità. criterio dellid quod interest, prima importante evoluzione della clausola di risarcimento aquiliana, venne introdotto indicava il "valore della cosa che aveva per il proprietario" Gaio, nel II secolo d C contribuia sviluppare la base di questa prima clausola generale risarcitoria, definendo il concetto stesso di danno "si plus dominus capiat darmulem id quoque aestimanu" Con $i$ Severi 12 concezione del danno viene definitivamente ridefinita come perdita patrimoniale differenza tra simazion originaria e quella che si è venuta a creare a seguito dellillecito, non attribuendo rilevanza alcuna al danno morale. Dal Digesto emersero git element -i giuristi francesi insegrano - della creazione del Code Civil del 1804, un quadro di presuppostid determinan i fondamenti di quello che le codificazioni moderne designeranno come istituto della responsabilità civile, vale a dire: la colpa la funzione mista della responsabilità (sanzionatoria e risarcitoria), eil danno. E' ancora da riportare che molte regole giuridiche sono state successivamente caricate di significato morale ed etico: l'altenum non laedere - per esempio - indica un principio generale ed astratto 
di ritenere la colpa, appunto concretizzante l'iniuria, intesa come negligenza del danneggiante, quale presupposto imprescindibile della responsabilità ${ }^{12}$.

Proprio in riferimento a questa interpretazione di iniuria ruota tutta la problematica attorno al concetto stesso di colpa: se, cioè, con il termine iniuria si indicasse solo un comportamento non iure o contra ius ovvero se, diversamente, si includesse anche la colpa quale elemento indispensabile per il delinearsi delle fattispecie di cui alla lex Aquilia. In particolare, come ricorda il romanista Schipani, si sarebbe fatto riferimento alla colpa, quale elemento determinante dell'illecito civile, solo nel momento in cui veniva superata "l'essenzialità del requisito del damnum corpore corpori dato", in quanto "condotta ed evento potevano verificarsi in momenti diversi". Ciò comportava che "si sarebbe presentata la necessità di precisare, in rapporto al criterio della responsabilità, sulla base di quali altri presupposti il soggetto fosse tenuto" e, conseguentemente, la colpa venne individuata in tutti quei casi di "ignoranza, debolezza, imperizia, negligenza, disavvedutezza, imprudenza"13.

E' sicuramente molto istigante e centrata la ricostruzione fatta da Alvino Lima, nella sua opera più classica ${ }^{14}$, in riferimento al dibattito sull'essenzialità del requisito della colpa ai fini della configurazione della risarcibilità prevista dalla lex Aquilia. Secondo lo stesso, coloro ${ }^{15}$ che considerano la colpa quale elemento fondamentale di tale illecito, fonderebbero il loro assunto nelle parole di Ulpiano, e cioè nell'espressione latina in lege A quilia et lerissima culpa venit ${ }^{6}$ : secondo cui, per l'applicazione della lex era, dunque, sufficiente la minima negligenza del danneggiante.

$\mathrm{E}^{\prime}$, quindi, inevitabile dover evidenziare la tradizionale ricostruzione operata da Jhering ${ }^{17}$, forgiatore del dictat "kein Übel ohne Schuld", ossia "nessuna responsabilità senza colpa", in base al quale nel sistema di diritto privato romano, trovava fonte il precedente storico della colpa, quale elemento appunto "da sempre" legato al concetto di illecito civile.

Diversamente, altra dottrina ${ }^{18}$ sostiene, ai fini dell'applicazione della lex Aquilia, 1 non essenzialità dell'elemento colpa, almeno ad origine. Tale dottrina parte, infatti, dalla considerazione secondo cui l'espressione in lege Aquilia et levissima culpa venit sarebbe, di fatto, il frutto di una interpolazione. La colpa sarebbe stata introdotta, quale elemento costitutivo,

12 SCHIFANI, Sandto. Responsabilità ex lege Aquilia, Criteri di imputazione e problema della colpa. Torino: Utet, 1968, p. 1 ess, ritiene che a tal conclusioni la pandettistica sia pervenuta nostrando di ignorare la divergenza esistente nel testo della legge in cui non si fa tiferimento all'elemento della colpa, ma a quello dell'iniuria. Tale contrasto è risolto da HASSE, Johan Christian. Die culpa des römischen Recht, 1938, p.8 ss. nel senso che il terrmene culpa assumerebbe un duplice significato: primo, di carattere ordinario, quale "infrazione di una norma qualsiasi", ed il secondo, di natura giutidica, inteso come "condotta illecita". Da questa duplice accezione discenderebbe la fungrbilità dei due ternumi, al punto di poter parlare indifferentemente di damnum iniuria datum o dench 13 SCHIPANI, Sandro. Op. Cit.

15 Tra classicicitati da Alvino Lima GIRARD, Paul Frédénc. Manuel élémentaire de droit romain. Paris 1906, MAY, Gaston, OP cit: JHERTNG Rudolf von "De la fate en droit privé", in Éndes complémentaires de lesprit de droit romain, trad fr de Meulenaere Paris 1880 .

Meulenaere. Paris, 1880.
16 Digesto 9.2.44.1. Ulpiano 42

17 JHERING, Rudolf von.. Das Schuldmoment in römischen Privatrecht. Leipzzig, 1867, p. 155 ss.

18 Alvino Lima cita BETTI, Emilio. Diritto Romano. Parte generale. Padova. 1935; COZZI, Mario. La responsabilità civile per danni da cose. Padcva: Giuffrè, 1935: MAZEAUD, Leon e Henri. Tratte theorique et pratique de la responsabilte civit délictuelle et contractuelle, t I, Ge éd. Paris: Éditions Montchrestien, 1965 solo in un secondo momento e per opera dell'interpretazione. Alcuni autori, tra cui spicca l'italiano Venezian ${ }^{19}$ che addirittura cerca di sintetizzare in chiave di responsabilità oggettiva il sistema privato romano ${ }^{20}$, inducono a sostenere la coesistenza di ipotesi di responsabilità senza colpa ${ }^{21}$ e ipotesi di responsabilità per colpa

Nonostante l'istigante dibattito sulla natura della responsabilità da illecito nel diritto romano, è comunque da evidenziare come, in realtà, sia stato il giusnaturalismo francese del XVII sec. ad aver meglio definito ed isolato il concetto di colpa ${ }^{22}$. La dottrina tradizionale ${ }^{23}$ è infatti concorde nell'attribuire a Domat ed a Pothier il merito di aver attuato la vera svolta in materia di responsabilità civile, $\mathrm{e}$, conseguentemente, aver contribuito al consolidamento della concezione "classica" della colpa, quale elemento imprescindibile del concetto stesso di responsabilità civile.

In qualche modo, $i$ due giuristi francesi possono dunque essere considerati i padri della teoria "classica" della colpa, intesa quale fondamento della clausola generale di responsabilità. La costruzione teorico-dottrinale da essi operata venne, infatti, poi adottata dal Code Civil francese del $1804 \mathrm{e}$ da tutte le altre codificazioni che, dal modello francese, trassero ispirazione. In particolare, Domat, pur continuando a descrivere dettagliatamente le varie fattispecie di illecito, in conformità alla tradizione romanista, che riconosceval'esistenza di tipiche e precise ipotesi di illecito, cercò di forgiare una nozione generale di fatto illecito. Pothier, nella stessa linea, insistette ulteriormente sulla descrizione di una fattispecie generale di illecito, cristallizzando la differenziazione tra i delitti e i quasi-delitti, approfondendo così il concetto di imputabilità ${ }^{24}$

Doma ${ }^{25}$ ebbe, dunque, il grande merito di aver introdotto una nozione ampia ed unitaria del fatto illecito, prendendo in esame non solo i fatti vietati espressamente dalle leggi, ma anche quelli che, in qualche modo, si ponevano in contras to con l'equità, l'onestà e il buon costume "anche se non vi siano leggi scritte che li prevedano" 2.

In sintesi, la necessità di individuare una "clausola generale" che abbracciasse tutta un'altra ampia area di ipotesi di fatti illeciti non normativizzati, è da derivarsi - sempre secondo Domat ${ }^{27}$ - alla necessità di un'ordinata conirivenza cinile. Questa ricostruzione fu determinante nell'elaborazione e nella stesura Code Civil francese ${ }^{28}$. Domat fu, dunque, il

19 VENEZIAN, Giacomo. Danno e risarcimento fuori dei contratti, 1884-1886 Roma 1918

20 GIORDANO, Francesca, Op. cit. p. 173

21 inffra 1 sistema della responsabilità ogrettiva dei fratelli Mazeaut

22 ALPA, Guido. Ull. Op.cit, p. 36 e ss

(2)

25 AL.PA, Guido. Op. cit. p.48 sostiene che Domat, pur analizzando e partendo dalle fonti del Digesto effettraa un'interpretazione ben diversa da quella operata dagli esponenti della scuola storica: infatti, non esegue una partizione tipica, ma una volta annunciata la definizione di taito illecito umperniata sul concetto di colpa esamma alcuni esempi di fatti illectí crvili.

26 MONATERI, Pier Giuseppe. Le fonti delle obbligazioni - La responsabilità civile, in SACCO, Rodolfo. Trattato di diritto civile. Torino: UTET 1998, p.135

$27 \mathrm{DOMAT}$, Les los civiles dans leur ordre naturel. 1, II, tit. VIII, in ID., Oeuv res complètes, nuova ed. a cura di REMY, J. t. I, Paris: Didot, 1835, p. 407 in ALPA, Guido, Diritto della responsabilità civile, Roma-Bari: Ed. Laterza, 2003 p. 48 ss ne IRTI, Natalino (Collana diretta da). Isturuzioni di Diritto. Serre monografica.

28 L.IMA, Alvino. Op. Cit. p. 28 
primo a distinguere con chiarezza le varie fattispecie di colpa ${ }^{29}$.

Pothier, non molto diversamente, riprese la ripartizione dei delitti e dei quasi delitti ${ }^{30}$ di chiara derivazione romanista, evidenziando come il delitto definirebbe il fatto attraverso il quale una persona, per dolo o per malevolenza, causa un danno o un torto ad un altro; diversamente, con il quasi delitto, si indicherebbe il fatto attraverso cui una persona, senza malevolenza, ma per una imprudenza non scusabile, causa torto ad un'altra. L'importanza di tale distinzione è fondamentale nel processo di consolidamento della colpa quale elemento che costituisce l'illecito ${ }^{31}$

\section{2) Insufficienza del concetto classico della responsabilità colposa}

Nonostante la colpa sia stata accettata dai vari sistemi di diritto come principio, o meglio, criterio di imputazione base su cui fondare la responsabilità da illecito civile, le me colpa quale unico meccanismo di imputazione di responsabilità.Le motivazioni annesse a colpa quale unico meccanismo di imputazione di rente sviluppo attengono principalmente a delle valutazioni non strettamente di carattere giuridico. La valutazione giuridica acquista significato in quanto conseguenza di una situazione sociale ben diversificata rispetto alle tradizionali relazioni tra individui.

L'avvento della rivoluzione industriale, le teorie economiche sul profitto, lo sviluppo economico senza precedenti hanno, di fatto, contribuito a che la riflessione attorno agli interessi valutati in ambito di responsabilità civile fossero oggetto di discussione e di revisione ${ }^{32}$. In particolare, la consolidata e particolarmente attenta dottrina francese, da un lato, e la rivitalizzata dottrina italiana, dall' altro, hanno contribuito significativamente ad una reinterpretazione degli interessi del danneggiante e del danneggiato, in chiave decisamente più "sociale" 33 .

L'interpretazione della responsabilità civile alla luce del principio della solidarietà porta ad una equa distribuzione dell'incidenza del danno. Interessante è notare come, in realtà, tale interpretazione vada a tutelare degli interessi che pur non essendo, molte volte, riconosciuti espressamente possono essere ricavati implicitamente dal l'intero sistema giuridico.

Il meccanismo a cui ci si riferisce per riconoscere e contemperare questi interessi è 29 Alpa nota, ancora, come Domat distingua la colpa in diverse sub-categorie: la colpa che comporta un reato ovvero un delitto la colpa per la violazione del rappotto contrattuale, colpa che na cattiva custodia di cose $O$ animal

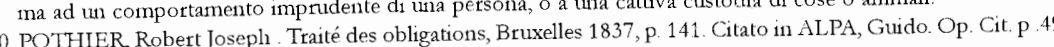

31 POTHIER, Robert Joseph. Op. cit.
32 Interessante è osservare come in realtà la crisi, Yinsufficienza del concetto tradizionale di responsabilità colposa non sia stato un fatto isolato, è da ricordare che anche in ambito contrattuale si parla di crisi dovuto alla massificazione delle relazion

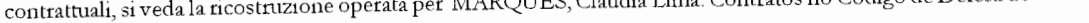
São Paulo: Editora Revista dos Tribunais, 2005 p. 163

33 Accanto al metodo tradizionale della responsabilità civile, quale quello dell'amministrazione del danno, la dottrina contemporanea è soltta apprezzare anche un approccio differente, la rilevanza economica. Infatti, a partire dagli anni Sessanta del XX sec. Pietro 'Trimarchi in Italia, Guido Calabresi e Richard Posner negli Stati Uniti (ip pric citati) hanno focalizzato 1 loro studi sulle tecniche di distribuzione del danno. Principalmente questa impostazione economica, nelle diverse "graduazion! $\mathrm{da}$ Calabresi "The cost of accidents" a Posner "Theories of economic reguta di prevenire il danno e non sia stata in grado di adottare la diligenza del buon padre di faniglia normalmente l'equità. Senza il ricorso all'equità, infatti, le disposizioni normative molte volte possono risultare inefficaci. Così come il giudice può rifarsi al principio dell'equità per attendere situazioni altrimenti non tisolvibili o limitatamente risolvibili, il legislatore può ispirarsi al principio dell'equità per regolamentare alla base tipi di situazioni difficilmente tutelabili dal raziocinio giuridico.

Il legislatore, dunque, per certi aspetti, coadiuva la posizione del giudice, indirizzandolo, attraverso la previsione legislativa di criteri di giustizia sostanziale, al riconoscimento dell'ingiustizia del danno, di modo che sia sempre garantita una risarcibilità, o al più, una indennizzazione da danno - appunto - ingiusto.

E' proprio nelle relazioni tra singoli individui e società che si riscontrano i casi più significativi in cui appare tutta l'insufficienza del concetto colpevolistico nella disciplina dell'illecito civile. L'esame degli interessi in gioco, dunque, prevede una sorta di bilanciamento: da un lato, l'interesse altrui minacciato da un comportamento, che normalmente è colposo, dall'altro, l'interesse che l'agente con quella condotta tenta di realizzare ${ }^{34}$. Gli interessi in gioco, il più delle volte, sono della più varia natura e quasi sempre non corrispondono ad una previsione normativa tipica: da qui, la necessità di una valutazione circa l'elemento colpevolistico del comportamento lesivo. Non sempre, infatti, la colpa corrisponde al un rischio giustificato, e viceversa ${ }^{35}$.

Il contemperamento degli interessi singolo individuo e società si inserisce esattamente in questo contesto, ossia nell'impossibilità di ricondurre - il più delle volte, per la mancanza di previsioni normative espresse o per irrazionalità giuridica - una previsione di rischio ad una valutazione conforme al criterio colpevolistico ${ }^{36}$. La conseguenza a questa insufficienza è, dunque, stata la presa in considerazione del concetto di utilità sociale - utilitas-circa l'esercizio dell'attività cui il rischio si riferisce.La valutazione degli interessi al fine di garantire la risarcibilità, o l'indennizzazione di una delle parti, non deve tener conto solo di aspetti di carattere economico, ma l'utilitas deve essere valutata in relazione a tutti gli aspetti della personalità dell'individuo ${ }^{37}$.

L'istituto della responsabilità civile, dunque, assume la funzione specifica di riequilibrare o riottemperare gli interessi tra le parti, nel caso in cui questi siano alteratio modificati in modo pregiudizievole per una sola delle parti: la responsabilità civile è intes a come giustizia sostanziale tra gli i singoli individui e tra individui e società (altro discorso varrebbe nei rapporti con la Pubblica Amministrazione, che generalmente difende interessi privilegiati). Infinite sono le funzioni della responsabilità perché infinite sono le situazioni che vengono a caratterizzare gli squilibri nella società.

In modo particolare, a partire da tempi molto recenti, in cui - appunto - lo sviluppo industriale e la crescita economica hanno contribuito a differenziare maggiormente casi di

34 TRIMARCHI, Pietro. Illecito in Enciclopedia del diritto, vol. XX, Milano 1970, pp. 103-104

35 TRIMARCHI, Pietro. Op cit, pp. 103-104

MELLO DA SILVA, Wilsor Responsabilidade sem culpa. São Paulo: Saraiva 1974 134 sulh socializzazione del diritto Inindividual ststo giuridico

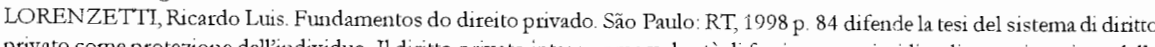
e protezione dell'nindividuo Il dritito privato inteso come volontà di farsi norma giuridica di organizzazione della 
ingiustizia sostanziale, la funzione reintegrativa o di giustizia sostanziale può ed è stata garantita solo da una oggettivazione della responsabilità civile, attraverso un onere probatorio meno gravoso o attraverso l'estensione dell'area risarcibile ${ }^{38}$. L'oggettivazione della responsabilità civile esige la distribuzione del rischio, al fine di poter garantire un continuo contemperamento degli interessi e un equo bilanciamento dei danni. L'attribuzione all'imprenditore del rischio d'impresa comporta dunque la razionalizzazione del mercato, attraverso la creazione di norme che tutelano la sicurezza: il rischio deve essere a carico di chi trae dall'attività svolta un vantaggio economico.

La responsabilità oggettiva ha dunque la funzione di regolare e rendere sostanzialmente più giusto il contemperamento degli interessi, laddove una attività che realizza un vantaggio individuale non sia proporzionale alla sua dannosità ${ }^{39}$.

\section{B) Dal principio della responsabilità per colpa alla teoria del rischio e il principio} della responsabilità oggettiva.

\section{1) La socializzazione del rischio}

L' innarrestabile processo di indistrializzazione, la moltiplicazione smisurata delle nuove pratiche tecnologiche, le nuove scoperte, il fenomeno dell'addensamento della popolazione nei grandi centri, la creazione di modeme centrali elettriche, tutto ciò che viene a determinare un costante miglioramento nelle condizioni di vita della popolazione comporta conseguentemente un' aumento proporzionato dei rischi.

Il concetto di colpa soggettiva, in ambito della responsabilità civile si dunque è dimostrato insufficiente e ciò a causa delle condizioni economico-sociali dettate dai grandi eventi che hanno e stanno caratterizzando la nostra epoca, oltre a motivi di ordine filosoficopolitico e giuridico, conseguenti al declino dell'individualismo e della naścita della socializzazione. Accanto alla tradicionale figura della colpa si è dunque fatta strada una nuova teoria, quella del rischio, che non rappresenta una figura eccezionale di responsabilità, ma, diversamente, si incastra "parallelamente" nella figura dell'illecito extracontrattuale.

La socializzazione del rischio offre la possibilità di una indennizzazione sempre garantita, senza che si presenti la necessità di un internento di nessuno, introducendo nel diritto un criterio più equitativo di distribuzione della giustizia. La socializazione dei rischi risponde alla logica di una maggiore giustizia sociale, e viene incontro alla realtà quotidiana degli eventi, che vede il passaggio da una forma di tutela del danno alla persona ad una tutela del danno all'intera collettività.

2) Responsabilità oggettiva: dall'imputazione per rischio-profitto all'imputazione per rischio-creato.

La nascita delle teorie del rischio solitamente vengono ricondotte ad una epoca storica ben determinata: ci si riferisce alla fine dell'Ottocento, inizio del Novecento. Si inizia, appunto, a discutere attorno a queste tematiche proprio in occasione di quel movimento dottrinario, sviluppatosi principalmente in area francese ed in area italiana, chiamato socialismo giuridico ${ }^{40}$. Si tratta di un particolare periodo in cui le conseguenze di uno sviluppo industriale senza precedenti iniziarono a mutare il corso delle relazioni sociali, economiche e giuridiche tra individui, e soprattutto tra individui ed imprese. Il socialismo giuridico, dunque, fu la risposta "sociale e giuridica" ad un mutamento avvenuto principalmente sul un piano economico.

Da parte francese, Saleilles, Josserand, Sauzer, Sainctelette, da parte italiana, Coviello, Barassi teorizzano il "fallimento" della colpa, nel senso che sviluppano, in ambito di illecito civile, tendenze e criteri orientati su una concezione della responsabilità del tutto dissociata dal concetto di colpa.

Le teorie del rischio vengono solitamente distinte in teorie del rischio-profitto e teorie del rischio-creato. In realtà, come evidenzia Lima ${ }^{41}$, originariamente ci si riferiva alla teoria del rischio integrale, e solo successivamente si passò a considerare la bipartizione tra teoria dell'atto anormale, difesa da Ripert, e la teoria del rischio.

La prima prevedeva la nascita di ipotesi di responsabilità quando l'attività esercitata da un agente fosse di tipo "anormale" nell'uso della proprietà: veniva in questo modo allargata la possibilità di rivalersi sul proprio vicino, nei rapporti specifici di vicinanza tra fondi.

In realtà, questa teoria non ebbe molto seguito, dal momento che fu molto criticata per vari aspetti, ma soprattutto per il fatto di celare un comportamento colposo dell'agente, e ricadere comunque nella previsione colpevolistica dell'illecito civile ${ }^{42}$. La seconda, ossia la teoria del rischio, è oggetto, a sua volta, di una bipartizione, o meglio di una vera e propria evoluzione ed integrazione. La teoria del rischio può, infatti, essere così ripartita e analizzata: da un lato, la teoria del rischio profitto, dall'altro, la teoria del rischio creato ${ }^{43}$.

La prima parte dal presupposto secondo cui l'attività di impresa, proprio perché finalizzata al conseguimento del maggior profitto, deve, allo stesso tempo, assumersi i rischi che da questo tipo di attività possono derivare. Ecco dunque la socializzazione della responsabilità: chi consegue un profitto da una attività non può considerare di fare ricadere i rischi sulla società stessa, ubi emolumentum, ibi onus. In particolare, dapprincipio, si cominciò a discutere in merito a queste tematiche riferendosi principalmente a quelle attività considerate pericolose, in quanto nocive alla salute degli individui, per poi arrivare ad abbracciare ogni attività di impresa. Saleilles evidenzia come il rischio diventa cosi parte integrante delle spese generali che un 'impresa deve sopportare ${ }^{44}$.

40 Responsabilità oggettiva per danni e responsabilità per tischio di impresa dov rebbero essere considerati concetti ben distint atteso che per valutare il rischio di impresa ci si dovrebbe rapportare alle teorie sulla distribuzione dei costi e dei profitti. Mentre a responsabilita lato sensu trova, infatt, la sua causa giustificatrice nella reintegrazione del patrimonio del danneggiato, i BESSONE OP it in

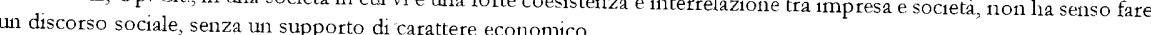
41 LIMA Alvino Op citza un supporto di carattere economico.

MAZEAUD Hennet T.

43 MAZEAUD, Henti et Léon, et TUNC, André. Op cit

44 SALEILLES, Raymond. La responsabilité du fait des choses devant la Cour Superieure du Canada, in Rev. Trim dr. Civ 1911 pp. 48 e segg, come esaminata da ALPA, Guido. Op. Cit. p. 290. 
La teoria del rischio profitto venne dunque affiancata, per così dire, da una evoluzione della stessa, ma che per i caratteri che presenta se ne differenzia, e prende il nome di teoria del rischio creato. Si tratta di una teoria più ampia e comprensiva anche della teoria del rischio profitto, e che consente di applicare criteri di responsabilità oggettiva anche a casi in cui non si sia in presenza di un' attività di impres $a^{45}$.

La nuova teoria evidenzia dunque il principio secondo cui chiunque impiega fonti di rischio deve sopportarne le conseguenze negative: non può essere considerato giusto ed equo quando la vittima che non ha contribuito alla creazione dei rischi sopporti le conseguenze negative prodotte dall'esercizio di tale attività. La teoria del rischio creato, senza dubbio, ha fissato le basi per la nuova concezione della responsabilità extracontrattuale oggettiva o senza colpa. Le teorie del rischio rispondono ad una logica di carattere sociale, la loro finalita è dunque quella di compensare, o meglio, procedere alla riparazione dei danni, seguendo il solo criterio obiettivo della valutazione, o meglio, della proporzionalità che caratterizza la relazione danno-beneficio.

\section{II- Esperienza comparativa tra il sistema di Civil Law e il sistema di Common Law:} modi differenti di introduzione della responsabilità oggettiva.

\section{A) I modelli di Civil Law:}

\section{1) La responsabilità colposa e la responsabilità oggettiva: Codice Civile francese}

\section{e Codice Civile italiano.}

Nel diritto francese

Il Code Civil francese del 1804 rappresenta sicuramente l'esempio più tipico, se non addirittura l'esempio più paradigmatico, delle legislazioni moderne che accolgono il principio della responsabilità per colpa. La clausola generale della responsabilità civile è disciplinata dagli artt. 1382 e 1383 CC, i quali sintetizzano il risultato degli studi e degli insegnament sulla colpa e sulla classificazione dei "delitti e quasi delitti" di Domat e di Pothier ${ }^{46}$.

L'art. 1382 CC così recita, "tout fait quelionque de l'bomme, qui cause à autrui un dommage, oblige celui par la faute duquel il est arrivé, à le réparer". La clausola è quindi affiancata e ampliata da un'altra importante clausola, quella stabilita all' art. 1383 CC che statuisce: "Chacun est responsable du dommage qu'il a causé non seulement par son fait, mais encore par sa négligence ou par son imprudence".

In realtà, nonostante la paradigmatica e tradizionale ricostruzione "colpevolisticosoggettiva" della responsabilità civile ${ }^{47}$, così come è possibile evincere anche da una immediata lettura degli stessi articoli sopra menzionati, la dottrina comparativa, in riferimento ai tre elementi costituenti la responsabilità dommage, faute e lien de causalité, critica l'utilizzazione di alcune espressioni ritenute non sufficientemente chiare ${ }^{48}$ : in particolare, l'espressione "dommage".

45 ALPA, Guido. Op. Cit. p. 291

$46 \mathrm{v}$. intra

47 LEVI, Giulio Responsabilità civile e responsabilità oggettiva. Milano: Giuffrè. 1986, p. 53

48 ZWEIGERT Konrad; KÖTZ Hein. Einfühnung in die Rechtsvergleichung auf dem Gebiete des Privatrechts. Tübingen Mohr, 3.Auflage, 1996, p. 619
Ci si domanda, infatti, se includa la definizione di danno morale, se tratti solo di un'offesa personale o se siano oggetto di compensazione anche altri interessi economici ${ }^{49}$.

Allo stesso modo, vi è una ricca e consolidata dottrina che si è occupata. dell'interpretazione del termine "faute", che costituisce il cardine attorno cui ruota tutto il sistema soggettivis tico della colpa, o, nello specifico, dei cosiddetti "quasi delitt"' 50 . Si tende, infatti, distinguere due diverse situazioni che possono essere de finite dal concetto di faute $e^{51}$. ossia, nella violazione di un precetto che definisce un comportamento da rispettare nella doppia concezione, da un lato, di volontà di causare danno (faute délictuelle), dall'altro, di comportamento criticabile secondo il principio generale del buon padre di famiglia (faute quasi-délictuelle) - reasonable man of ordinary prudence.

Nonostante le pur criticate ${ }^{52}$ espressioni utilizzate dal codice per definire gli elementi propri dell'istituto della responsabilità gli autori sono concordi nel definire che il Code Civi si fondi sul principio colpevolistico ${ }^{53}$. Tuttavia, la dottrina più recente e la stessa giurisprudenza della Cour de Cassation partendo dall'esame dell'art 1384 CC e segg. sembrano non essere della stessa opinione. L'art 1384, par. 1, CC così recita: "On est responsable non seulement du dommage que l'on cause par son propre fait, mais encore de celui qui est causé par le fait des personnes dont on doit répondre, ou des choses que l'on a sous sa garde". Si tratta chiaramente di una responsabilità per fatto altrui. Ma non si tratta del solo caso, il Code Civil, agli artt. $1385^{54}$ e $1386^{55} \mathrm{CC}$, dispone un'altra presunzione di responsabilità in capo al proprietario di animali e al proprietario di edificio rovinoso.

Interessante è notare come l'art. 1384, par. $1^{\circ}$, CC escluda la previsione di responsabilità solo nei casi di cas fortuit o force majeure, ossia quando il fatto dannoso è determinato da un evento esterno il cui verificarsi non dipende, in alcun modo, dalla volontà dell'agente.

Appare chiaro che considerate le poche ipotesi in cui è possibile riscontrare un caso fortuito o una forza maggiore, la quasi totalità delle ipotesi in cui viene ad essere applicato il 1384 , par. $1^{\circ}, \mathrm{CC}$ determina la previsione di una responsabilità indipendente da qualsiasi negligenza dell'agente.

Nonostante l'evidente e sopra richiamata previsione codicis tica di cui agli art. $1382 \mathrm{e}$ $1383 \mathrm{CC}$, nasce il problema di come considerare queste ipotesi di imputazione di responsabilità.

La ricostruzione di Josserand aiuta evidenziando che la ragione per la quale l'art. 1384 , par. $1^{\circ}, \mathrm{CC}$ trasferisce la responsabilità ad altro soggetto risiede nel fatto che la società risponde ad un interesse sociale oggettivo, ossia in quello di garantire una compensazione

49 ZWEIGERT Konrad, KÖTZ Hein. Op. cit. p 620

50 AUBRY, Charles Marie Barbe Antoine. RAU, Charles Frederic. Cours de Droit Civil Franç̧ass, d'après la méthode de Zachariae

51 VINEY, Geneviève; JOURDAIN, Patrice. Les conditions de la responsabilité, in Traité de drott civil Paris, LGDI, 1998 53 ZWWEIG

54 Art. 1385 CC "I "Le pad; KÖTZ Hein. Op. cit. p. 663 ss.

que l'animal a causcoprétaire d'un animal, ou celui qui s'en sert, pendant qứil est à son usage, est responsable du dommage 5 Att. 1386 CC "Te proptét que l'animal fût sous sa garde, soit qu'il fût égaré ou échappée.

du défaut d'entretien ou par le vice de sa constrsponsable du dommage causé par sa nuine, lorsqu'elle est arrivée par une suite 
del danno: si tratta della cosiddetta dottrina del rischio creato $^{56}$. Josserand, in tal modo, riconosce che la semplice presunzione di responsabilità, che solo superficialmente richiama il principio colpevolistico, in realtà nasconde ipotesi di responsabilità senza colpa, in cui, cioè il soggetto agente viene ad essere liberato in casi del tutto eccezionali ${ }^{57}$.

Questa impostazione è solo una delle risposte dottrinarie all'esame del 1384, par. $1^{\circ}$, CC, la lettura ancora dominante è quella dei fratelli Mazeaud ${ }^{58} \mathrm{e}$ di André Tunc che difendono l'idea secondo cui la responsabilità civile nel diritto francese rimane caratterizzata dalla predominanza del principio colpevolistico, indipendentemente dalle varie previsioni di presunzione di colpa.

\section{Nel diritto italiano}

Il codice civile italiano del 1942 è, sénza dubbio, una creazione codicistica di chiara ed espressa influenza francese. Di conseguenza, lo stesso istitu to della responsabilità civile, che ammette esplicitamente la colpa quale elemento costitutivo dell'illecito, riflette la ricostruzione "soggettivista" operata dall'art. 1382 del Codice Civile francese. E' cosi possibile incontrare la presenza dell'elemento "colpa" già all'articolo "fondante" della stessa responsabilità civile del sistema codicistico italiano, ossia l'art. 2043 CC $^{59}$

Nonostante l'esplicito riferimento all'elemento colpa, è tuttavia possibile individuare, già da un'analisi più approfondita dell'art. 2043 CC, come il binomio illecito civile - colpa non sia, in realtà, così stretto e, soprattutto, chiuso ad altre ricostruzioni.

Rescigno evidenzia che, se non fossero possibili altre ricostruzioni, si dovrebbe ipotizzare un errore del legislatore, nell'aver usato la parola "fatto", anziché "atto", quale causa determinante dellillecito. Da una lettura complessiva della norma, - chiarisce poi 56 Perla dhéorie du risque créé si veda anche SALEILLES, Raymond. Op. Cit., pp. 48 e ss. “.il rischio diventa parte integrante delle spese generali ed è l'industria che ne sopporta il carico, con tripattizionle propotzionale tra coloro che cooperanio, gl tul sotto

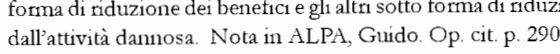

dall'attività dannosa. Nota in ALPA, Guido. Op. Cit. p. 290.
57 Della stessa linea VINEY Genevieve; JOURDAIN, Patrice. Op. cit. p. 531

58 Come nota Alvino Lima, nel suo già più volte citato studio sulla colpa e sul rischio, lo studio dei fratelli Mazeuad esamina molto attentamente P'istituto della responsabilità civile. La precisazione è d'obbligo dal momento che l'opera dei Mazeuad appare essere indispensabile per meglio comprendere la differenziazione esistente tra il concetto di responsabilità obiettiva $e$ responsabilità per colpa obiettiva. Essi partono dalla considerazione che la valutazione della colpa può essere fatta in concreto $e$ in abstracto e ciò porterebbe ad una distunzione tra colpa obiettiva e colpa soggettiva. I Mazeaud distinguono, poi, una imputabilità del tutto prescindente dalla colpa, quella determinata ed espressione delle teorie sul rischio, da altrí due tipi di imputabilità che dipendono, invece, dalla presenza della colpa, che però può distinguersi in colpa in abstracto, e colpa in concreto. Diversa è dunque la concezione obiettiva della responsabilità. nel senso di un crterio di inputazione che non st fondamenta sulla condotta dell'autore del danno: prescinde dal comportamento volontario dell'agente, sia esso conseguente di una valutazione un abstracto o in concreto. E questa la concrenzzazione della teonia del nischio. Nel caso esanmiato dal Mazeaud si taatta invece di distinguere lesume delh colp a saber essere accertata preste quando la valutuzione prescinde dal lato psicologico dell'autore, e accosta il comportamento dell'agente ad un tipo astratto (culpa iil abstracto), il bonus pater familiae al di là della critica mossa a tale ricostnuzione l'opera dei Mazeaud è sicuramente

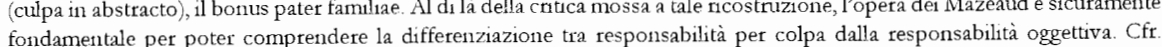
MAZEAUD, Henty et Léoni; TUNC, André. Op. Cit

Art 2043 CC "Qualunque fatto doloso o colposo, che cagiona ad altri un darno ingiusto, obbliga colui che ha commesso is fatto a risarcire il danno" apparirebbe preferibile l'utilizzazione del termine "atto", anziché "fatto", in quanto si integrerebbe meglio con la forte connotazione di volontà e consapevolezza, cui allude la norma stessa nel caratterizzare l'azione dell'autore dell'illecito ${ }^{60}$.

Si tratterebbe, dunque, di una sorta di apertura a posizioni interpretative, per lo più, svincolate dalla tradizionale ricostruzione "soggettivistica" dell'illecito civile italiano.

Seppur tale rivisitazione interpretativa dell'art. 2043 CC, sia molto stimolante e, sicuramente, degna di un più dettagliato approfondimento, ciò che, tuttavia, non può essere messo in discussione è l'espresso riferimento all'elemento "colpa" quale circostanza fondante dell'illecito.

I dubbi, dunque, sulla ricostruzione soggettivis tica della responsabilità civile italiana sembrerebbero cosi risolti, atteso il chiaro riscontro di cui all'art. $2043 \mathrm{CC}^{61}$, se non fosse per gii articoli seguenti. In particolare, il codice disciplina alcune particolari figure di illecito in cui sono previsti dei criteri di imputazione differenti rispetto al generale criterio "soggettivo" rappresentato dall'art. 2043 CC.

$\mathrm{Ci}$ si riferisce alle ipotesi di responsabilità "per fatto altrui" ${ }^{\text {, }}$, alle ipotesi di responsabilità "per danni cagionati da cose in custodia o da animali" ${ }^{\prime \prime}$, alle ipotesi di responsabilità per danni cagionati "da rovina di edificio o dalla circolazione di veicoli senza guide di rotaie" alla responsabilità per danni "da attività pericolose"

Si tratta di ipotesi considerate dalla dottrina tradizionale ${ }^{66}$ eccezioni a quella regola

Si discute sul termine "fatto" usato all'art. 2043 CC. Il legislatore ha infátti adottato la dicitura "fatto" anziché " "atto". legislatore sembra alludere all'evento dannoso come ad una conseguenza del fatto dell'uomo, dal monento che allude alla consapevolezza e alla volontà, ossia all'elemento volitivo. Pettanto, l'utulizzazione di tale termine concretizza il sospetto che l'apertura porta al termine "fatto", uno svincolo da posizioni colpevolisticle del'"llecito Cfe, RESCIGNO Pietro hand di diritro privato Napoli Jo late 1977 . 688 ess. di distopith

62 Att. $2047 \mathrm{CC}$ "In caso di danno cagionato da persona incapace d'intendere o di volete il tisarcimento è dovuto da chie tenuto alla sorveglianza dell'incapace, salvo che provi di non aver poturo impedire i fatto Nel caso in cui il daunegrato non abbi potuto ottenere il risarcimento da clie tenuto alla sorveglanza, il gudice, in conside. Nione delle condizioni economiche delle

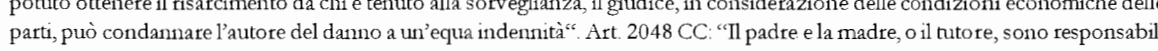
del danno cagionato dal fatto illecito dei figli minori non emancipatio o delle persone soggette alla tutela, che abitano con essi. La stessa disposizione si applica all'affiliante. I precettori e coloto che insegrano un mestiere o un'arte sono responsabili del danno cagionato dal fatto illecito dei loro allievi e apprendisti nel tempo in cui sono sotto la loro vigilanza. Le persone indicate dai commi precedenti sono liberate dalla responsabilità soltanto se ptovano di non avere potuto impedire il fatto"; Art. 2049 CC: "I padroni e i committenti sono responsabili peri danni areecati dal fatto illecito dei loro domestici e commessi nellesercizio delle incombenze a cui sono adibitic

63 Art. 2051 CC: "Ciascuno e responsabile del danno cagionato dalle cose che ha in custodia, salvo che provi il caso fortuito"; Art $2052 \mathrm{CC}$ : "Il proprietario di un animale o chi se ne serve per il tempo in cui lo ha in uso, è responsabile dei danni cagionat dall'anirnale, sia che fosse sotto la sua custodia, sia che fosse smarrito o fuggito, salvo che provi il caso fortuito"

64 Art. 2053 CC. "I proprietario di un edificio o di altra costruzione è responsabile dei danni cagionati dalla ioro rovina, salvo che provi che questa non e dovuta a difetto di manutenzione o a vizio di costruzione"; Art. 2054 CC. "Il conducente di un veicolo di aver fatto hutto il possitilie per evitare il damno. Nel caso di scontro tra veicoli si presume fin a prova contraria, che ciascuno dei conducenti abbia concorso ugialmente a produre ll durno subito da singoli veicoli vece, 'usufntriction la circolazione del veicolo è avverulta contro la sun voloutì In ogri caso le persone incicate dai cormi precedert cho responsabili dei danni derivati da vizi di costruzione o da difetto di manutenzione del veicolo"

65 Art. 2050: "Chiunque cagiona danino ad altri nello svolgimento di un'attivitia peticolosa, per sua natura o per la natura dei mezzi adoperati, e tenuto al risarcimento, se non prova di avere adottato nutte le misure idonee a evitare il dannoc. 66 DE CUPIS, Adriano. Il danno. Milano: Giuffrè 1979 
generale contenuta all'art. 2043 CC. In realtà, l'evoluzione dottrinale e giurisprudenziale attorno a questo dibattito può considerarsi ancora non definita, attesa la complessa articolazione di indirizzi che si sono avvicendati dall'adozione del codice.

Sicuramente, l'interpretazione più classica e tradizionale è data da De Cupis ${ }^{67}$, il quale partendo da una serie di ragioni che verranno ad essere esaminate da qui a poco, afferma che, in realtà, la regola deve essere considerata sempre quella dell' adozione del principio Jheringhiano "nessuna responsabilità senza colpa", e che, evidentemente, i casi di responsabilità oggettiva, rappresentati da questi differenti criteri di imputazione, sarebbero davvero così esigui da dover per forza di cose essere considerati del tutto eccezionali. Solo alcuni fatti non colposi nota - produrrebbero responsabilità ${ }^{68}$.

Le fattispecie di cui agli artt. 2047, 2048,2050,2054, $1^{\circ}$ e $3^{\circ}$ comma CC - osserva ammettono prova liberatoria "salvo che proir di non aver potuto impedire il fatto" ${ }^{16} \mathrm{O}$ "se non prova di aver adottato tutte le misure idonee a eirtare il danno", ovvero "se non provia di aver adottato tutte le misure idonee a enitare il danno" ${ }^{\prime \prime 1}$ e ciò configurerebbe, dunque, una sorta di "presinnzione semplice" di colpa che, di fatto, darebbe origine solo ad un'inversione dell'onere della prova.

Per quanto attiene all'art. 2049 CC, che non ammette prova liberatoria, non si tratterebbe comunque di un criterio "svincolato dalla colpa" dal momento che la responsabilità deriverebbe da "colpa da altro soggetto" legato da un rapporto di subordinazione ${ }^{72}$. Gli art. 2051 e 2052 CC concretizzerebbero una sorta di "presunzione assoluta di colpa" superabile solo attraverso la prova di un elemento estraneo, il caso fortuito ${ }^{73}$.

Attesa la riconducibilità di tutte questi criteri di imputazione al generale criterio della colpa, De Cupis, e la dottrina tradizionalista tutta, riduce a due sole ipotesi "eccezionali" $i$ casi

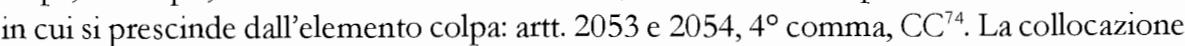
codicistica e un'esame ben più approfondito di tali norme lascerebbe intendere che l'art. 2043 CC rappresenti in realtà la regola generale della responsabilità, mentre i successivi articoli rappresentino solo delle eccezioni ${ }^{75}$.

Diversamente, la dottrina più moderna ${ }^{76}$ ha modificato integralmente non solo l'interpretazione di tale collocazione all'interno del codice, ma addirittura la stessa concezione di responsabilità civile ${ }^{77}$, proprio per ricomprendere quelle particolari ipotesi di

67 DE CUPIS, Adriano. Op. cit

1993

69 Artt. 2047 e 2048 CC

70 Art. $2050 \mathrm{CC}$.

71 Art. 2054 CC.

73 DE CUPIS, Adriano. Op. cil

74 “ l'obbligo del detto comportamento è obiettivamente ineseguito ogniqualvolta anche a prescindere da riproverole negligenza, la necessità attività preventiva non è completamente svolta..." Da DE CUPIS, Adriano. Op. cit

75 BESSONE, Mario. La responsabilità civile. Milano Giuffre, 2001

76 Anche la stessa Giurspprudenza ha operato una riadattamento del concetto di colpa di cui all' art. $2043 \mathrm{CC}$, in senso più oggettivo, non più dunque in termini puramente soggettivi, al fine di tutelare le esigenze di protezione dell'uomo moderno. Cfr. VISINTINI, Giovarna. Trattato breve della responsabilita civile. Fatti illeciti. Inadempimento. Danno nisarcibile. Padova: CEDAM, 1996

Cfr. TRIMARCHt, RODOTA, BIANCA ma anche CALABRESI. Citati da CASTRONOVO, Carlo. La lluova responsabilita civile. Milano: Guuffrè, 1997. Cfr. BIANCA, Cesare Massimo. La responsabilità. Vol. V, in Diritto Civile. Milano: Giuffrè, 2003 danneggiamento che si verificano indipendentemente da un comportamento volontario del soggetto e garantire, conseguentemente, una tutela concreta all'individuo di fronte ad una società "nuova", industriale e consumistica.

Ecco, allora che, come ricostruisce Trimarchi ${ }^{78}$, esisterebbero in realtà due diverse ipotesi di responsabilità, una per colpa e una oggettiva, non più in rapporto di gerarchia, in cuil'imputazione per colpa rappresenta la regola mentre quella oggettiva l'eccezione, ma di vera e propria parità ${ }^{79}$. Trimarchi differenzia le attività umane, che chiama attività biologiche, dalle attività economiche ed organizzate, e afferma che la responsabilità oggettiva risponderebbe ad una esigenza di tutela legata a danneggiamenti determinati dalle attività economiche e organizzate, mentre la tradizionale responsabilità per colpa coprirebbe tutte le altre ipotesi tradizionalmente "biologiche"

E', dunque, possibile affermare che, sebbene nel codice italiano non si parl esplicitamente di responsabilità oggettiva, essa sia comunque riconosciuta e utilizzata dalla dottrina e dalla giurisprudenza.

2) La responsabilità colposa e la responsabilità oggettiva: Codice Civile tedesco e Codice Covile Brasiliano.

Nel dirittmo tedesco

Il codice civile tedesco (BGB) non ha seguito il grande modello francese ma ha assunto caratteristiche e peculiarità proprie. Gli studi romanistici e la storica scuola del diritto di Savigny hanno, per così dire, modellato la codificazione tedesca. La codificazione tedesca, diversamente dalle codificazioni francese e italiana (in cui è presente una clausola generale di responsabilità), è dunque più legata alla tipizzazione delle fattispecie di illecito ${ }^{81}$, e riconosce espressamente la figura della responsabilità oggettiva, prescindendo anche dalla tradizionale distinzione classica tra responsabilità contrattuale ed extracontrattuale (da qui, la ragione della trattazione differenziata $)^{82}$

La legge regolamenta le fattispecie di illecito, prevedendo una relazione obbligazionale speciale di indennizzazione - da delikte - quale conseguenza di un comportamento illecito (artt. da 823 a 853 BGB). In sintesi, ogni volta che un comportamento umano pregiudica illecitamente un altro soggetto determina il sorgere di un'obbligazione di indennizzazione peri danni causati.

Fondamentalmente, l'obbligazione di indennizzazione sorge laddove l'autore del danno illecito abbia agito con colpa, intesa nel senso di dolo o negligenza. La responsabilità per rischio (gefährdungshaftung) è invece configurata come ipotesi di responsabilità senza colpa, sempre in ambito di rapporto obbligazionale nato da delikte, o deliktsrecht $t^{83}$.

78 TRIMARCHI, Pietro. Rischio e responsabalilià oggettiva. Milano Guffrè̀, 196

79 TRIMARCHI, Pietro Op cit

80 TRIMARCHI Pietro Op cit

81 FERRARI, Franco. Tipicità e atipicità del fatto illecito, in G.ALGANO, Francesco (a cura di) At̉ante di Dirito Privato Comparato Bologna Zanichelli, 1999 p. 139 "q a mancata previsione dei fatti illeciti guale fonti di obbligazicne secondo tahumi avvalorerebbe la tesi della tipicità, in quanto questa sarebbe sufficiente a tivelare la scarsa rilevanza dell 1 istituto che altro non può significare se tonn previsione di tipicità?

82 LEVI, Giulio. Op. cit., p. 55

83 SHAPP, Jan Einführnung in das Bürgerliche Recht. München: GmbH, 2003, p. 152 
Il BGB individua in tre articoli $(823 \$ 1,823 \$ 2$ e $826 \mathrm{BGB}) \mathrm{i}$ criteri per determinare l'illiceità del comportamento umano e dunque i criteri che fondano l'obbligazione di indennizzazione ${ }^{84}$

L'art. $823 \$ 1$ prevede l'indennizzazione laddove la violazione sia relativa a beni giuridici e diritti ben individuati e nominati. L'art. $823 \rrbracket 2$ prevede l'obbligo di indennizzazione in caso di violazione di una legge posta a protezione delle persone in generale. L'art. 826 prevede l'obbligo di indennizzazione quando il pregiudizio è contro i buoni costumi ${ }^{85}$. Interessante osservare, come ricorda Levi, che ancora nel secondo progetto del BGB, appunto in relazione alla norma del 823 , si discuteva se tutti i beni giuridici fossero oggetto di protezione e, dunque, se l'estensione dell'obbligo di risarcimento avesse dovuto riguardare anche quelle violazioni senza colpa ${ }^{86}$. La Commissione della Camera dei Deputati, non avendo approvato il progetto relativamente all'inclusione di queste violazioni senza colpa nel 823 BGB, optò, diversamente, per l'adozione di disposizioni particolari e specifiche rispondenti al principio secondo cui il rischio di determinate attività deve accollarsi a chi le esercita $^{87}$.

Nel diritto tedesco, dunque, le ipotesi di responsabilità civile senza colpa hanno avuto una prima disciplina espressa in leggi specifiche. $\grave{E}$, per esempio, il caso della legge $n$ 25 del 1938 che stabilisce la responsabilità assoluta delle società ferroviarie per i danni a persone o cose oggetto di trasporto, o la legge del 7 luglio 1971 in materia di attività industriale.

Larenz evidenzia che le ipotesi di responsabilità senza colpa, nel diritto tedesco, devono essere considerate eccezionali, così come dimostrato dal ricorso alla regolamentazione per mezzo di leggi speciali (ci si riferisce principalmente alla responsabilità delle ferrovie o alla responsabilità aerea). Larenz, in realtà, chiarifica che solo quando queste leggi si fondano sul principio della responsabilità per rischio possono riconoscersi ipotesi di responsabilità a colpa oggettiva, dal momento che prescindono da ogni riferimento ad un atteggiamento soggettivo dell'agente. Larenz, dunque, differenzia queste ultime ipotesi, di responsabilità per colpa oggettiva, da quei casi di responsabilità senza colpa, ove in realtà - chiarisce - si tratta di presunzioni di colpa del soggetto cui viene imputata la responsabilità, come nel caso della responsabilità dei padroni e dei committenti ${ }^{88}$

In sintesi, con le leggi speciali si è regolata la responsabilità da Delikte o "extracontrattuale" senza colpa, ossia la responsabilità per rischio, in cui colui che trae profitto deve assumersi la responsabilità per i danni che tale attività determina.

E' questo il principio della giustizia distributiva: colui che trae profitto da un'attività deve assumersi i danni che quella attività determina, secondo il principio riassunto dal brocardo latino secondo cui cuius commoda eius et incommoda. Tuttavia, non sempre il diritto tedesco

84 SHAPP, Jan. Op. Cit. p. 153

85 ESSER. Josef. Schuldrecht. Karlsruhe. 1968. p. 43. considera che attraverso il 826 BGB passano tutte le fattispecie atipiche di illecito.

86 LEVI, Giulio. Op. cit., p. 55

87 LEVI, Gullio Op, cit., p 55

88 LARENZ, Karl. Lehrbuch des Schuldrechts. München: Beck, 1964, p. 451

89 Si usa qui un termine improprio, atteso che - come gia ante argomentato - il diritto tedesco non accoglie la separazione tradizionale tra responsabilità contrattuale ed extracontrattuale. configura ipotesi di responsabilità oggettiva, a meno che non si tratti di attività pericolosa $\mathrm{O}$ rischiosa, solo quando si tratti di casi tassativamente previsti dalla legge. In base a quanto stabilito all' art $823 \$ 1 \mathrm{BGB}$, la responsabilità fa riferimento non solo al bene personale della vita, della salute, ecc. ma anche al diritto soggettivo assoluto, che ricomprende i diritti della personalità, il diritto al nome, il diritto all'immagine ecc.. Dunque, per il diritto tedesco chiunque che, con la propria attività, incide illegittimamente su interessi altrui, che devono appunto essere intesi nel senso di diritto soggettivo assoluto, deve assicurare sicurezza e protezione contro i rischi che ne derivano (Verkehrssicherungspflichten) ${ }^{90}$.

Quando la lesione non è prevedibile ed evitabile e, dunque, non valutabile ai fini di un comportamento colposo, la dottrina è concorde nel ritenere che non si ha responsabilità ex art. $823 \$ 1 \mathrm{BGB}, \mathrm{ma}$, al più, potrebbe darsi ai sensi del $823 \$ 2 \mathrm{BGB}^{91}$. Dottrina più recente ritiene che anche se è possibile avere una infrazione di una legge senza colpa ${ }^{92}$, l'obbligazione di indennizzazione di cui all' art. $823 \$ 2 \mathrm{BGB}$, sorgerebbe esclusivamente in caso di colpa e, dunque, in presenza di almeno un comportamento da considerare negligente ${ }^{93}$.

Zweigert e Kötz ${ }^{94}$ sostengono che la responsabilità oggettiva, o senza colpa, nel diritto tedesco è stata introdotta esclusivamente attraverso leggi speciali non incorporate nel codice, laddove - chiariscono - il legislatore ha voluto presumibilmente richiamare il principio della colpa dell'illecito civile o della responsabilità da delitto ${ }^{95}$.

\section{Nel diritto brasiliano}

Nonostante l'evidente e, più volte, riconosciuta influenza italiana e portoghese nella redazione del codice civile brasiliano del 2002, appare preferibile accostare - limitatamente e strettamente alla ripartizione sistematica del presente studio - il modello brasiliano a quello tedesco. Ciò per una ragione molto semplice: il diritto civile brasiliano, al pari di quello tedesco, riconosce ${ }^{96}$ senza alcun tipo di incertezza o di titubanza, anche se attraverso l'utilizzo della legislazione speciale, ipotesi di responsabilità oggettiva ${ }^{97}$.

Il diritto civile brasiliano, infatti, ammette esplicitamente due tipi di responsabilità

90 LEVI, Giulio. Op. Cit. p. 57

91 LEVI, Giulio Op. Cit. p. 57

92 FERRARI, Franco. Op. Cit. p. 142 " con riferimento all' ambito di applicazione di tale norma occorre avvertire che essa opera una Beweiserleichterung, ossia facilita al danneggiato la prova, in quanto questo dovrà solamente provare la violazione de Schutzgesetz, non anche il nesso di casualità vigendo in questi casi una presunzione. Inoltre, il comma in esame dà al danneggrato nelle ipotesi in cui il schutzresetz sin qulificabile come nouma

93 SHAPP, Jan. Op. Cit.p. 180

94 ZWEIGERT Konrad; KÖ'TZ Hein. Op. cit. p. 684 e ss.

96 PONTES DE MIRANDA, Francisco Cavalcanti. Tratado de direito privado. São Paulo. Editora Revista dos Tribunais, 1984 Vol 53 p. $77-78$ un iferimento al codice civile del 1916, ora abrogato e sostituito dal CC del 2002 cosí argomenta: "No sistern do diretto civil brasileiro, a responsabilidade pelo ato, positivo ou negativo, de outrem, é transubjetiva. Não adotamos a responsabilidade dita objetiva"

97 E' nuttavia da evidenziare, che, differentemente dal BGB, il codice civile brasiliano ammette la nota ripartizione responsabilità contrattrale - extracontrattuale, tornando ad evidenziare quella continuità di impostazione italiana (e in questo caso, francoitaliana) che è possibile riscontrare in tutto il codice civile. 
civile $^{98}$ : la responsabilità civile soggettiva, e la responsabilità civile oggettiva ${ }^{9 \%}$. La prima ha valenza generale ${ }^{100}$ e permea tutti i principali istituti del diritto civile brasiliano, rappresentando, dunque, quella continuità codicistica di ispirazione franco-italiana, di una responsabilità principalmente fondata sulla teoria classica della colpa ${ }^{101}$.

La seconda, ovvero la responsabilità cosiddetta oggettiva, in quanto, appunto, prescinde dall'esame e dalla prova della condotta dell'agente (elemento psicologico), riflette le attuali tendenze del diritto a voler sanzionare $o$, in qualche modo, controbilanciare tutti quei comportamenti che, per certa natura, costituiscono di per se una limitazione o un pregiudizio all'esercizio di diritti a danno della comunità, e rispondono, quindi, alla logica e all'sspirazione della già, più volte, supra citata teoria del rischio.

L' is titu to della responsabilità civile ne diritto brasiliano è di natura riparatoria ed è costruito sul concetto di atto illecito, sia esso contrattuale sia esso extracontrattuale ${ }^{102}$. Per atto illecito si intende tutto ciò che in qualche modo è contrario alla norma giuridica. Il codice civile brasiliano definisce all'art. $186 \mathrm{del} \mathrm{CC} \mathrm{del} 2002$ la disciplina dell'atto illecito (diversa e distinta è la previsione dell'atto illecito relativo all'inadempimento contrattuale, prevista all'art. 389 CC): "aquele que, por ação ou omissão voluntária, negligencia ou imprudência, violar direito $e$ causar dano a outrem, ainda que exclusivamente moral, comete ato ilicito "mos.

Ugualmente, nel campo della responsabilità contrattuale, è possibile individuare ipotesi di responsabilità a carattere soggettivo, con riferimento a tutte le componenti soggettive ${ }^{104}$ del negozio giuridico ${ }^{105}$, e ipotesi di responsabilità a carattere oggettivo, con riferimento ai casi di cui agli articoli 421 e $422 \mathrm{del} \mathrm{CC/2002,} \mathrm{relativi} \mathrm{all'aspetto} \mathrm{oggettivo} \mathrm{del}$ negozio giuridico.

Dunque, per parlarsi di responsabilità civile o extracontrattuale nel sistema di diritto civile brasiliano ci si deve riferire agli artt. 186 e $927 \mathrm{CC}^{106}$. Quest'ultima previsione codicistica è d'uopo, al fine di riconoscere un'ulteriore classificazione dell'illecito civile, ossia la ripartizione tra responsabilità soggettiva e responsabilità oggettiva, di cui già supra argomentato.

98 PONTES DE MIRANDA, Francisco Cavalcanti Op Cit VOL 53, p. 35 . O direito das obrigações tem de considerar os fatos ilicitos absolutos, como a ofensa ao corpo humano, aos animais pertencentes a outrem e aos bens inanimados, eos fatos ilícitos relativos, como a infração da clausula contratual. Nos fatos ilícitos, quer absolutos, quer relativos, estão os atos ilícitos, como os atos-fatos ilícitos e os fatos ilícitos stricto sensu. E há responsabilidade sem ilicitude do ato

99 NERY JUNIOR, Nelson e DE ANDRADE NERY, Rosa Maria. Código Civil Comentado, Săo Paulo: RT, p. 608

100 MOREIRA ALVES, José Carlos. A responsabilidade extracontratual e seu fundamento: culpa e nexo de causalidade, in Est. Oscar Corrêa, in. 5 , p. 200, in NERY JUNIOR - DE ANDRADE NERY, Op. cit, p. 608.

101 CAVALIERI FILHO, Sérgio. Responsabilidade civil 110 novo Código Civil Revista do Direito do Consumidor 48, p. 80 ss., ou - dez. 2003.

02 GOMES, Orlando. Introdução ao direito civil Rio de Janeito: Forense, 1971 e GOMES, Orlando. Contratos. Rio de Janeiro: Foretise, 1973

103 Traduzione libera perl' italiano. "colui che, per azione o omissione volontaria, negligenza o imprudenza, viola il diritto e causa un danno altrui, anche se esclusivamente motale, commette un atto illecito". Art. 186 CC

104 Cri. art. 389 e segs. CC/2002; arts de 138 a 140 CC $/ 2002$

105 Il codice civile brasiliano del 2002 accetta la definizione di negozio giuridico Livro III, Dos fatos jurídicos, Tín Il I Do negócio juídico. Conffonta: JUNQUEIRA DE AZEVEDO, Antôtrio. Negócio Jurídico. São Paulo: Saraiva, 2002.

Interessante è osservare come il legislatore brasiliano abbia voluto differenziare il sistema della responsabilità da illecito civile
dalla responsabilità da illecito o inadempimento contrattuale, definendo per i due diversi istituti diverse definizioni di atto illecito. Si può durqque osservare la utilizzazione del modello italiano
La prima parte del 927 CC recita: "aquele que, por ato ilicito causar dano a outrem, fica obrigado a repará-lo $b^{107}$ ”. Il codice civile brasiliano all'art. 927 CC stabilisce, dunque, l'atto illecito come fonte di responsabilità e come fonte dell'obbligazione di indennizzo ${ }^{108}$.

Quanto all'istituto della responsabilità oggettiva, esso trova esplicito riferimento codicistico al paragrafo unico dell'art. $927^{109} \mathrm{CC}$ del 2002 e fonda il proprio assunto sulla già nota ed esaminata teoria del rischio.

La responsabilità oggettiva è, come già è stato analizzato, un tipo di responsabilità che nasce indipendente da un comportamento di carattere psicologico. Si tratta di una responsabilità che determina l'obbligo di riparare il danno causato in tutti quei casi previsti dalla legge $\mathrm{o}$, in generale, quando l'attività normalmente esercitata dall'autore del danno implica, ex se, un rischio circa la possibilità di veder realizzato il diritto altrui (art. $927 \mathrm{CC} /$ 2002 par. ún.).

E' questa una forma di responsabilità che decorre inevitabilmente da quella insufficienza della concezione tradizionale della colpa soggettiva, incapace, o meglio, non in grado di saper rispondere a tutte quelle esigenze economiche, sociali e politiche che la vita moderna e contemporanea impongono.

Il codice civile brasiliano del 2002, dunque, sulla linea di tale interpretazione, riconosce esplicitamente ipotesi di responsabilità obiettiva. Da una parte, prevedendo tutte quelle ipotesi che, in qualche modo, possono essere riconducibili alla previsione della clausola generale, o meglio, del "paragrafo unico" dell'art. 927 CC/2002, quando cioè si tratta di tutte quelle ipotesi in cui è esercitata un'attività che, solo per il fatto di essere compiuta, crea, per sua natura, un pregiudizio (ad esempio, le "classiche" attività pericolose), dall'altra, tichiamando precise e determinante ipotesi previste dalla legge.

Secondo una ricostruzione fatta dal giurista Francisco Amaral ${ }^{110}$, queste ultime possono essere cosi di seguito annoverate: danos resultantes da ruína de edificio ou construião (art 937 CC/2002); queda ou lancamento de coisa em lugar indevido (art. 938 CC/2002); acidentes de trabalbo (Lei n. 8.213, de 24 de julho de 1991); acidentes em estradas de ferro (Lei n. 2.681, de 01 de dezembro de 1912) e, per analogia, os aidentes de transportes coletivos, na navegação aérea (Lei n. 7.565 de 19 de dezembro de 1986); dano ambiental (Lei n. 6.938 de 31 de agos to de 1981, art 14, \$1); dano nuclear (CF, art. 21, XVIII, c, Lei n. 6.453, de 17 de outubro de 1977, art. 4);

107 Traduzione libera per 1'ítaliano: "colui che, con atto illecito causa un danno ad altri, è obbligato a tipararlo"

108 L'art. 927 CC stabilisce quale fonte dell’obbligazione di indennizzo o ripatazione anche un'altta previsione normativa, quell relativa a quanto previsto all'art. $187 \mathrm{CC}$ relativa all'abuso di diritto "também comete ato ilicito o titular de un direito que, ao exercê-lo, excede manifestamente os limites impostos pelo sen fim econòmico ou social, pela boa fé ou pelos bons costumes" In italiano, "commette, allo stesso modo, atto illecito il titolare di un diritto che, nell' esercitarlo, eccede manifestamente l limit imposto dal fine economico e socrale, dalla buona fede o dar buon costum". Interessante e osservare come si riscontra

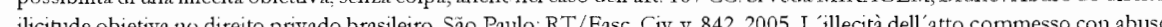
del dititto è di natura oggettiva, presciande dal dolo e dalla colpa: non é necessario la coscienza di eccedere i limitt fissati dalla norma nei principi in essa trascritti ma é sufficiente oltrepassare i limitt in essa fissatt.

109 Atr 927 CC 2002 parágrafo único "Haverá obrigação de reparar o dano independentemente de culpa, nos casos especificado em les, ou cuando a atividade normalmente desenvolvida pelo autor do dano implicar, por sua natureza, risco para os direitos

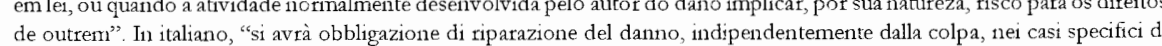

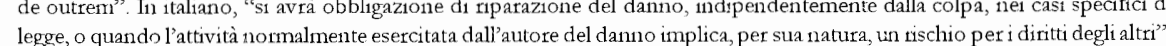
110 AMARAL, Francisco. Direito Civil, Introduçăo. Sảo Paulo. Renovar, 2006, pag. 558 
prestạ̧ão de serviço público (CF, art. 37, \$6; CC art. 43); e, in materia di consumo, fato do produto de serviço (Lei n. 8.078, de 11 de setembro de 1990, arts. 12, 14).

\section{B) I modelli di Common Law:}

1. Esperienza inglese: Torts e Tort of negligence.

Nel sistema anglosassone, la responsabilità da illecito è tradizionalmente basata sul principio della tipicità, law of torts ${ }^{171}$. Tuttavia, dagli anni trenta, alle tipiche figure dei torts venne ad affiancarsi il generale principio del tort of negligence $e^{172}$, applicabile ad ogni ipotesi di illecito a danno di terzi ${ }^{113}$

Questo affiancamento, o meglio, questo mutamento a favore di una clausola atipica di responsabilità, fu la conseguenza dell'adozione di una decisione da parte della House of Lords del 1932, relativamente al caso Donoghue versus Stevenson ${ }^{14}$, in materia di protezione del consumatore ${ }^{115}$. Siffatta decisione aprì le porte al processo di atipizzazione delle ipotesi di responsabilità nel diritto inglese ${ }^{116}$. Interessante è notare come l'apertura all'atipizzazione del tort sia stata determinata da una controversia avente ad oggetto il trasferimento di un bene in una relazione produttore - consumatore.

L'imputabilità del terzo, appunto il produttore, normalmente estraneo alla relazione contrattuale col consumatore finale - considerati i diversi passaggi che il prodotto subisce prima di arrivare al destinatario finale -, evidenzia come una delle parti, appunto il consumatore, sia considerato "comunque più debole", e dunque, nella posizione di necessitare di una tutela integrale maggiore. La scelta di estendere il giudizio di negligence anche ad un soggetto terzo, rifiutando in siffatto modo il giudizio sulla componente psicologica dello stesso, concretizza una responsabilità oggettiva o senza colpa.

L'atipizzazione dell'illecito, attraverso l'imputabilità per negligence ${ }^{117}$, há dunque allargato le ipotesi di responsabilità, includendo tra il criterio di imputabilità atipico anche la responsabilità senza colpa, con la precisa finalità di tutelare l'integrità fisica e di perseguire la risarcibilità, in ogni caso, della lesione. Il termine negligence - al pari di quanto si verifica in un sistema di ciril law - letteralmente indica incuria o assenza di attenzione. Il termine negligence è dunque usato in senso generale per caratterizzare la condotta in relazione ai diversi tipi di

111 CANE, Peter. The Anatomy of Tort Law Oxford: Hart Publishing., 1997

112 FRIDMAN, Modern Tort Case, London 1968. Afferma che le ipotesi di illecito da coipa non sono mai chiuse $e$, in modo particolare, per quanto attiene allla negligenza, si creerebbe la possibilità di una estensione indefinita

113 Ricostruzione operata da LEVI, Giulio. Op. Cit. p. 5

114 Donoghue v Stevenson, A.C 562,1932

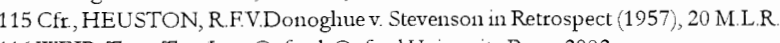

117 GALLO, Paolo Tipicitie atipicita dell illecito in comnon law in GALGANO. Francesco (a cura di Op CIt, p 144 " "Il tort of negligence ha wizito a svilupparsie ad assumere wa configurazione autonoma solo molto recentemente, diciamo a partise dallla fine del secolo scorso, anche pruma ss parlava di negligence, ma unicamente con rifermento all 'elemento soggettivo di certi torts. Basti pensare che ancora nei primu decenni del ventesino secolo si discuteva se il negligence fosse un tort vero e cert torts. Basta pensare che ancora nei prinu decenni del ventesinno secolo si discuteva se il negligence fosse un tort vero e
proprio o ooltanto un elemento costitutivo degli altri torts. Il nuovo tort ... si andava espandendo addiritura nell' area prima coperta da altri torts... tanto da assumere un noolo di primo piano nel sistema iuglese degli illeciti. Grazie a questa espansione il tort of negligence non puo più considerarsi alla stregua di un qual siasi altro rimedio inglese, ma é diventato in materia di colpa il rimedio per eccellenza, ovvero l'azione delittuale per antonomasia da intentarsi in presenza di un danneggiamento colposo alle cose o alle persone? illecito $^{118}$. Tuttavia, la stessa denominazione viene ad assumere una connotazione particolare. Negligence acquista un senso molto tecnico: indica, infatti, quelle condizioni in presenza delle quali un soggetto incorre in responsabilità, senza avere l'intenzione di causare alcuna lesione o danno. Tali condizioni sono indicate come: la presenza di un dovere di condotta (duty of care), la violazione dello stesso dovere di condotta (breach of duty of care) e un danno o un lesione, conseguenza di tale violazione (damage or injury) ${ }^{110}$.

Interessante è osservare come, nel diritto anglosassone, si sia sviluppata una graduale "apertura" del duty of care, e, appunto, della responsabilità del terzo. Infatti, il criterio tradizionale di imputazione della responsabilità non prevedeva che il terzo potesse rispondere in alcun modo nei confronti di una parte contrattuale, dal momento che era appunto estraneo alla relazione contrattuale. Con questa decisione, si andò ad estendere l'imputabilità anche a terzo, limitatamente - almeno dapprincipio - alle ipotesi di trasferimento aventi ad oggetto beni pericolosi.

Pioniere furono le Corti americane che con la decisione MacPhersomv' Buick Motor del 1916, aprirono la possibilità di una rivisitazione di tale principio. Solo poco dopo, la famosa e sopra citata decisione Donoghue r. Stevenson aprì le porte all'applicazione e all'estensione ad altre e più generali ipotesi di questo principio anche in Inghilterra.

Si è soliti sottolineare come questo tipo di responsabilità prescinde dall'esistenza di un vero e proprio nesso causale in concreto, laddove invece è presente un nesso causale, per così dire, solo astratto. Pur mancando una relazione causale, ai fini dell'imputabilità, è da ritenere che sia comunque sempre possibile riferirsi a colui che ha posto in essere e ha provveduto alla distribuzione del prodotto, il produttore ${ }^{120}$. Le pronunce anglosasson partono dal principio secondo cui il produttore può non aver utilizzato - traducendo letteralmente - la diligenza (negligence) o, semplicemente, l'avvedutezza necessaria per la creazione del prodotto, ben consapevole del fatto (essendo questa la finalità della immissione del prodotto nel mercato) che il prodotto sarebbe stato, prima o poi, oggetto di un uso da parte di un quaisiasi consumatore ${ }^{121}$. Non si tratta infatti di un illecito contrattuale, atteso che, nella maggior parte dei casi, il produttore non è parte contrattuale dell'utente finale. In questo modo, pur non esistendo, per la maggior parte dei casi, una relazione diretta tra produttore e consumatore finale (relazione causale concreta), l'integrità fisica di quest'ultimo viene comunque ad avere la precedenza, in quanto valore giuridicamente anteposto a qualsiasi valore di tipo patrimoniale.

2. Esperienza nordamericana: tutela del consumatore come veicolo di generalizzazione della responsabilità oggettiva.

Stati Uniti e Gran Bretagna sono accomunati dal fatto di appartenere alla stesso sistema di diritto, il common law. Nonostante il sistema giuridico sia lo stesso, i due paesi più

118 Siveda nota 110

119 ZWEIGERT Konrad; KÖTZ Hein Op. cit. p. 611 e ss

120 ALPA, Gưdo e BESSONE, Mario. Il problema dell'atipicità dell'illecito, Milano: Giuffrè 1995. p. 43

121 La responsabilità è dunque doryta ad una regola generale, ossia quella di tenere un compo tbe costituisce negligence 
tradizionali di common law presentano alcune differenziazioni: in modo particolare, con riferimento all'oggetto del presente studio, ossia in riferimento alla materia della responsabilità oggettiva o strict liability.

L'istituto della "responsabilità civile" è conosciuto nei paesi di common law come il diritto dei torts, e la responsabilità civile senza colpa, appunto strict liability.

Il sistema della responsabilità civile per colpa, che costituisce la regola per le tradizionali codificazioni nel sistema di civil law, viene ad essere, secondo le esigenze, approssimativamente convertita in un sistema di responsabilità oggettiva o strict liability ${ }^{122}$. In particolare, il sistema del common law statunitense si caratterizza per il fatto di accettare espressamente l'istituto della responsabilità oggettiva, senza arrivare a derivarla, o meglio, "estrarla" dalla codificazione del generale principio della responsabilità per colpa.

La famosa decisione delle corti inglesi Rylands versus Fletcher del $1868^{123}$ venne accettata e utilizzata anche dalle corti americane per estendere il generale principio della strict liability a particolari attività pericolose. La responsabilità oggettiva se originalmente caratterizzava esclusivamente ipotesi di responsabilità per rischio è andata allargando le proprie previsioni anche ad attività non di stretto rischio, come all'infortunio sul lavoro, in caso danni provocati all'ambiente, nel caso di fabbricazione di prodotti dannosi o per quanto attiene alla protezione del consumatore ${ }^{124}$

Il diritto americano e i casi trattati nelle corti americane descrivono una situazione altamente caratterizzata dalla presenza di ipotesi di responsabilità oggettiva. Interessante è osservare che gran parte di questi casi, in cui appunto si fa riferimento alla responsabilità senza colpa, riguardano la protezione del consumatore ed i prodotti difettosi ${ }^{125}$.

Nel sistema di diritto statunitense, dunque, il riconoscimento della responsabilità oggettiva è stato assunto quale principio normale, non eccezionale, diversamente dalle tradizionali scuole di civil law $w^{126}$

Le attività che tradizionalmente sono considerate pericolose non costituiscono tutte le attività rischiose: esistono infatti varie attività che possono essere considerate rischiose in senso lato dal momento che la finalità della tutela mira a coprire 1 'integrità fisica della persona o appunto del consumatore. Interessante è evidenziare anche come le corti americane tendono ad estendere la protezione della garanzia contrattuale sul bene anche al consumatore finale, alla sua famiglia e addirittura ai terzi entrati in relazione con lui. A volte, giustificano tali garanzie argomentando che le stesse deriverebbero direttamente dal bene in quanto tale, altre volte, focalizzano il bene oggetto della tutela nella relazione diretta tra produttore e consumatore finale.

Addirittura, si giunge ad evidenziare che il diritto americano, nel caso in cui un

122 ZWEIGERT Konrad; KÖTZ Hein Op cit. p. 672 ess

123 Cfr. Rylands versus Fletcher del 1868 trattato da SHUGERMAN, Jed Fandelsman. The Floodgates of Strict Liability. Bursting Reservoirs and the Adoption of Fletcherv Rylands in The Gilded Age in The Yale Law Journal, Vol. 110, No. 2 (Nov., 2000), pp 333-377

124 LEVI, Giulio. Op. Cit. p. 58

125 Classico èi caso Escolaversus Coca Cola Bottling Co. of Fresno (California) 1944, 150, Pac 2d 436, dove si puó osservare come della salute umana

126 ZWEIGERT Konrad; KÖTZ Hein. Op. cit. p. 672 e 673 consumatore viene ad essere pregiudicato da un difetto obiettivo del prodotto, pur arrivando a riconoscere i principi contrattuali, l' ambito di applicazione della disciplina legale ricade nella materia del tort $\mathrm{O}$ in questo caso della strict liability in tort.

Nel sistema statunitense, la tendenza dell istituto della responsabilità oggettiva non è quella di sostituire quello della responsabilità per colpa, ma, diversamente, è quella di affiancarlo al fine di attribuire alle vittime di certi tipi di violazioni una difesa particolare, che altrimenti non potrebbe essere garantita a causa, per esempio, della difficoltà di fornire la prova. L'ipotesi più tipica, appunto dimostrata dalla sorprendente casistica delle corti americane, è l'esempio relativo alla posizione del consumatore, che senza alcuna colpa si trovi a subire un pregiudizio determinato da un problema attinente al bene o al servizio oggetto della relazione di consumo, senza adeguata tutela a riguardo. Proprio per questo, l'area relativa alla difesa del consumatore negli Stati Uniti è divenuta un campo di sviluppo ${ }^{127}$ e di affermazione sempre più importante della responsabilità oggettiva.

\section{Considerazioni finali}

L'evoluzione della società comporta inevitabilmente un continuo adeguamento del diritto. I rapporti che vengono a determinarsi tra gli individui necessitano sempre di strumenti attualizzati e modellati in maniera tale che la giustizia sostanziale sia sempre mantenuta in un continuo riequilibrio e bilanciamento delle posizioni giuridiche in gioco.

Gli istituti tradizionali, ormai millenari, dimostrano la grande affidabilità e certezza nella regolamentazione dei rapporti, ma, allo tempo stesso, possono mostrare le inadeguatezze di un sistema creato e pensato nella finalità di tutelare esigenze sociali ed economiche diverse. Il diritto, dunque, è inteso come un sistema vivo, muterole, flessibile chè, in qualche modo, reagisce di fronte alla continua evoluzione della società. Il presente lavoro tende ad evidenziare come si stiano sviluppando le risposte giuridiche alle richieste di una società, sempre più esigente e alla disperata ricerca di una tutela maggiore.

Nonostante i diversi sistemi di diritto, le diverse codificazioni, le diverse scelte legislative adottate si può assistere - almeno tra i sistemi di diritto oggetto del presente studio - che esiste, di fatto, una convergenza nell'adozione di scelte normative comuni. La responsabilità oggettiva, giustificata dalle teorie del rischio, rappresenta una sorta di traguardo nella disperata ricerca di una giustizia sostanziale per situazioni in cui gli istituti tradizionali non riescono a garantire un equo bilanciamento degli interessi. Le codificazioni, seppur restie all'abbandono degli insegnamenti tradizionali dimostrano un'apertura maggiore verso le nuove teorie, al punto da arrivare ad estrapolare nuovi concetti da vecchi dogmi. Colpa e rischio sempre più sono visti come criteri di imputazione paritari e non più in un rapporto di predominanza $O$ di alternanza, ancor che se ciò si verifica con l'adozione di previsioni legislative speciali, intente a tutelare queste nuove relazioni giuridiche. 